\title{
Increased Monocyte Chemiluminescence in Cystic Fibrosis Patients and in their Parents
}

\author{
WARREN E. REGELMANN, NORMAN M. LUNDE, PRISCILLA T. PORTER, AND \\ PAUL G. QUIE \\ Department of Pediatrics, University of Minnesota School of Medicine, Minneapolis, Minnesota 55455
}

\begin{abstract}
We examined the chemiluminescence response of peripheral blood monocytes from patients with cystic fibrosis (CF) and their asymptomatic parental carriers of the CF gene to three different types of stimulation. We found that monocytes from both patients and carriers have increased luminol-dependent chemiluminescence in the first $25 \mathrm{~min}$ after stimulation by adherence to glass. These results are consistent with the hypothesis that monocytes from both $\mathrm{CF}$ heterozygotes and homozygotes respond to adhesion with increased oxygen radical formation. The increased adherence-induced monocyte chemiluminescence of the parental carriers did not vary with age or length of exposure of the parents to a child with CF. Also, repeated exposure to medications and respiratory secretions of CF patients was not associated with an increase in adherence-induced monocyte chemiluminescence of their nonbiologically related caretakers. Thus, this observed increase in chemiluminescence is not simply secondary to the medications or respiratory dysfunction seen in the patients with CF. Patients with other types of obstructive lung disease did not show increased adherence-induced monocyte chemiluminescence. We conclude that increased early phase adherence-induced monocyte chemiluminescence occurs in patients with cystic fibrosis and the obligate carriers of the $\mathrm{CF}$ gene independent of environmental influences. (Pediatr Res 20: 619-622, 1986)
\end{abstract}

\section{Abbreviations}

CF, cystic fibrosis

PMA, phorbol myristate acetate

FVC, forced vital capacity

FEV , forced expiratory volume at $1 \mathrm{~s}$

CPM, counts per min

CL, chemiluminescence

$\mathrm{CF}$ is the most common lethal autosomal recessive disease trait found in the Caucasian population, occurring in approximately one of every 2000 live births. The carrier frequency has been estimated to be as high as 5\% (1). In the CF homozygote, the disease is characterized by chronic pulmonary disease accompanied by bacterial infection, pancreatic insufficiency associated with malabsorption and malnutrition, and an elevated sweat chloride level. CF heterozygotes appear to be clinically normal.

Received May 6, 1985; accepted February 18, 1986

Correspondence Dr. Warren E. Rengelmann, University of Minnesota. Box 47 Mayo Memorial Building, 420 Delaware Street, S.E., Minneapolis, MN 55455.

Supported by the American Lung Association Grant 141, the National Institutes of Health Grant HL 27355, the Minnesota Medical Foundation, and the Cystic Fibrosis Foundation Center Grant to The University of Minnesota. P.G.Q. holds The American Legion Research Professorship.
Although long sought, neither the basic biochemical abnormality nor the gene product of $\mathrm{CF}$ are known (2-5).

Increased oxygen uptake and mitochondrial electron transport activity have been reported in the skin fibroblasts of both $\mathrm{CF}$ homozygotes and heterozygotes (6). The current study was designed to examine whether peripheral blood monocytes, whose oxygen metabolism during phagocytosis involves both mitochondrial and nonmitochondrial pathways (7), would show differences among CF patients, their parents, and controls in oxygen radical production. As a sensitive indicator of oxygen radical production we studied the amount of luminol-amplified CL (811) generated when the purified peripheral blood monocytes of CF patients, carriers, and their age-sex matched controls were stimulated by opsonized zymosan, PMA, or by adherence to the sides of glass liquid scintillation vials (12).

\section{METHODS}

Human subjects. After informed consent, four groups of individuals were studied. Each individual was compared with a simultaneously examined age-sex matched control selected from the normal population. This study was approved by the Human Subjects Committee at the University of Minnesota.

Group one consisted of $15 \mathrm{CF}$ patients between the ages of 2 and 40 . All had elevated sweat chloride levels by the method of Gibson and Cooke (13). All had had evidence of pancreatic insufficiency. Most were regularly receiving bronchodilators, pancreatic enzyme replacement, and antibiotic therapies. All patients had their pancreatic insufficiency controlled at the time of study. FVC varied from 38 to $124 \%$ predicted (median = $67 \%), \mathrm{FEV}_{1}$ from 23 to $114 \%$ predicted $($ median $=39 \%) \mathrm{FEV}_{1} /$ FVC from 0.38 to 0.87 (median $=0.61$ ) and Brasfield scores (14) from 1 to 19 (median score $=12$ ) at the time of study.

Group two was comprised of 21 parents of $\mathrm{CF}$ patients, i.e. obligate CF carriers. Nine of these were parents of CF patients who were diagnosed within $1 \mathrm{wk}$ prior to testing. Thus, these individuals were obligate CF carriers who had less exposure to environmental factors associated with living with and caring for a patient with $\mathrm{CF}$ such as medications and bacterial flora common in the sputum produced by older patients with CF. These factors could conceivably alter the parents' monocyte CL.

Group three consisted of 11 people who routinely cared for CF patients but were biologically unrelated. Included were two respiratory therapists, four spouses of CF patients, one private day care attendant, and six foster or adoptive parents. These individuals were routinely exposed to $\mathrm{CF}$ environmental factors but were not known CF carriers.

To determine whether obstructive lung disease and the use of bronchodialators and antibiotics by these patients could alter $\mathrm{CL}$, a fourth group of 13 individuals without evidence of $\mathrm{CF}$ but with obstructive pulmonary disease were studied. Eight had chronic obstructive pulmonary disease with $\mathrm{FEV}_{1} / \mathrm{FVC}<0.80$ 
and chronic cough. Five had asthma with obstructive pulmonary disease significantly reversible by $\beta$-agonists.

Individuals with evidence of acute or chronic bacterial infection were excluded from groups two, three, and the control group. They were not excluded from group four. Individuals who had symptoms of acute viral infection or who were immunodeficient were excluded from the study.

Monocyte preparation. Monocytes were purified using preconditioned plastic flasks as previously described $(15,16)$. The resulting cells were at least $95 \%$ monocytes by nonspecific esterase staining and greater than $90 \%$ viable by the trypan blue exclusion method.

Fifty-one of the 67 adherence assays were performed without the technician knowing whether samples were from experimental or control individuals. Before identifying the donors, data pairs were rejected if the raw CPM for any given vial was less than two times the background levels, or if their coefficient of variation from duplicates exceeded $30 \%$.

Chemiluminescence assays. To measure zymosan-induced CL, $2 \times 10^{4}$ monocytes suspended in $1 \mathrm{ml}$ HBSS with $0.1 \%$ gelatin $(\mathrm{w} / \mathrm{v})$ were added to duplicate scintillation vials containing zymosan, preopsonized in pooled human serum for $10^{\prime}$ at $37^{\circ} \mathrm{C}$ and $1.3 \mu \mathrm{M}$ luminol in $4.5 \mathrm{ml}$ HBSS. Vials were counted in a Beckman LS100 scintillation counter using an open window and one photomultiplier tube set out of coincidence. They were counted approximately every $4.1 \mathrm{~min}$ until after the peak in light flux.

PMA-induced CL was measured in the same manner as zymosan-induced CL, but $2.2 \mu \mathrm{g}$ PMA in place of zymosan was added to vials containing $4.5 \mathrm{ml}$ HBSS with $1.3 \mathrm{mM}$ luminol.

To measure adherence-induced CL, $2.5 \times 10^{5}$ monocytes in 1 $\mathrm{ml}$ HBSS were added to vials containing $1.3 \mu \mathrm{M}$ luminol in 4.5 $\mathrm{ml}$ HBSS. Because the presence of protein can inhibit leukocyte adherence to glass (12), no gelatin was present in the reaction mixture. All vials had been dark-adapted, background CL levels had been recorded, and all monocytes were added in the dark. Time 0 was the time of addition of monocytes. Vials were counted approximately every 4.1 minutes in a Beckman LS100 liquid scintillation counter using an open window and one photomultiplier tube set out of coincidence. The vials were removed from the scintillation counter after $25 \mathrm{~min}$, rinsed once gently with HBSS, and the adherent cells counted by releasing the nuclei with Zap-o-globin II (Coulter Diagnostics). Results are expressed as peak scintillation CPM per adherent cell; the average of the duplicates is reported.

\section{RESULTS}

When opsonized zymosan was used as a stimulant, monocytes from $11 \mathrm{CF}$ patients exhibited similar $\mathrm{CL}$ to their matched controls [mean difference in CPM per cell $\pm 1 \mathrm{SEM}=3.3 \pm 1.6$, $\left.\mathrm{p}_{\alpha}(\mathrm{t})=0.065\right]$. Zymosan-induced CL from nine CF carriers also was not significantly different from controls [mean difference in $\mathrm{CPM}$ per cell $\left.\pm 1 \mathrm{SEM}=-0.19 \pm 0.87, p_{\alpha}(\mathrm{t}) \geq 0.30\right]$.

PMA-induced CL was slightly lower in monocytes from 11 CF patients than controls [mean difference in CPM per cell \pm 1 $\left.\mathrm{SEM}=-2.03 \pm 0.65, p_{\alpha}(\mathrm{t})=0.01\right]$. PMA-induced CL from nine $\mathrm{CF}$ carriers showed no significant difference when compared with controls [mean difference in CPM per adherent cell \pm 1 $\left.\mathrm{SEM}=0.87 \pm 1.10, p_{\alpha}(\mathrm{t})>0.30\right]$.

When allowed to adhere to glass liquid scintillation vials (Fig. 1 ), monocytes from the $11 \mathrm{CF}$ patients and $21 \mathrm{CF}$ carriers, generated significantly higher early CL than their respective matched controls (Fig. 2) [mean difference from control in peak CPM per adherent cell \pm 1 SEM: CF patients versus controls $2.70 \pm 0.51, p_{\alpha}(\mathrm{t})<0.001 ; \mathrm{CF}$ carriers versus controls $1.69 \pm$ $0.40, p_{\alpha}(\mathrm{t})<0.001$; Fig. 3]. The kinetics of the CL response during the first 25 min was similar in all groups. There was no significant difference in number of monocytes adhering at $25^{\prime}$ between $\mathrm{CF}$ and control pairs [mean difference $=-1.22 \times 10^{4}$

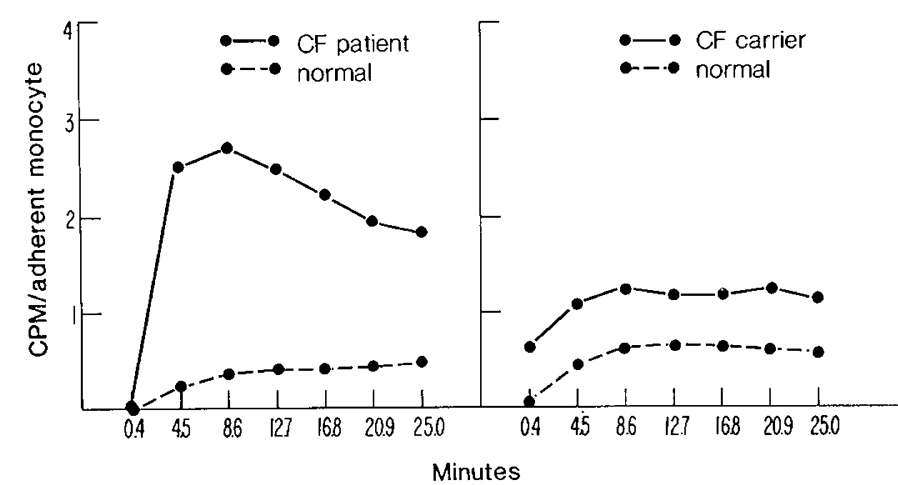

Fig. 1. Adherence-induced CL: a single experiment. Normal controls were age- and sex-matched to the CF patient and carrier. Glass adherence produced an early plateau or initial small peak occurring before $25 \mathrm{~min}$, followed by a gradual increase in light production per adherent monocyte. Discrimination between CF patients, carriers, and controls was obtained by comparing the initial peaks or the highest rate of light production within the first 25 min using a two-tailed paired $t$ test.

cells, $\mathrm{SEM}=1.2 \times 10^{4}, n=15, \mathrm{P}_{\alpha}(\mathrm{t})=0.33$ ]. Nor was there a significant difference in adherent $\mathrm{CF}$ carrier monocytes at $25^{\prime}$ compared to control [mean difference $=-0.73 \times 10^{4}$ cells, SEM $\left.=0.37 \times 10^{4}, n=21, \mathrm{P}_{\alpha}(\mathrm{t})=0.06\right]$. To control for environmental influences, nine of these carriers who were parents of newly diagnosed patients were separately analyzed and they too generated significantly higher CL [mean difference from control in peak CPM/cell $\left.\pm 1 \mathrm{SEM}=0.78 \pm 0.33, p_{\alpha}(\mathrm{t})=0.045\right]$. Moreover, when all carriers were analyzed there was no association between the difference in CPM from control and the length of time they were exposed to a CF patient.

Finally, monocytes from caretakers of CF patients who were not biologically related to them (group 3 ) showed no difference from matched controls in their adherence-induced CL [mean difference in peak CPM per adherent cell \pm 1 SEM $=0.02 \pm$ $0.39, p_{\alpha}(\mathrm{t})>0.30$, Fig. 3]. Similarly the presence of obstructive pulmonary disease in patients who did not have cystic fibrosis (group 4) did not increase the adherence-induced CL over matched control values [mean difference in peak CPM per adherent cell \pm 1 SEM $=0.26 \pm 0.28, p_{\alpha}(\mathrm{t})>0.30$, Fig. 3]. Interestingly, the five patients with asthma had a small but significantly decreased adherence-induced CL compared to their matched controls [mean difference in peak CPM per adherent cell \pm 1 SEM $\left.=-0.40 \pm 0.12 p_{\alpha}(\mathrm{t})=0.03\right]$ and none had CL above that of his matched control. In both of these groups the probability $(\beta)$ of not detecting a difference as large as that seen between CF carriers and controls was $\leq 10 \%$.

\section{DISCUSSION}

During phagocytosis, peripheral blood monocyte oxygen consumption increases dramatically $(7,17)$. This oxygen is rapidly converted into superoxide anion, hydrogen peroxide, and hydroxyl radical via one and two electron transfers to oxygen, with concurrent oxidation of NADH or NADPH (16). These oxygen products can be measured with high efficiency by luminoldependent chemiluminescence assays $(8-10,18)$. Luminol, however, will emit detectable photons whenever it is oxidatively dioxygenated (19). Thus, oxidants other than oxygen radicals, per se, might be responsible for the effects we observed. For example, peroxidases present in freshly isolated monocytes will likely produce $\mathrm{HOCl}$ from $\mathrm{H}_{2} \mathrm{O}_{2}$ and $\mathrm{Cl}^{-}$. The combination of $\mathrm{HOCl}$ and $\mathrm{H}_{2} \mathrm{O}_{2}$ significantly increases the $\mathrm{Cl}$ from luminol over that with $\mathrm{H}_{2} \mathrm{O}_{2}$ generating systems alone. Moreover, $\mathrm{OH}^{-}, \mathrm{O}_{2}^{-}$, and ${ }^{1} \mathrm{O}_{2}$ do not seem critical to the production of luminol $\mathrm{CL}$ by $\mathrm{HOCl}$ with $\mathrm{H}_{2} \mathrm{O}_{2}$ (20). The major alternative source to oxidase generated oxygen radicals and peroxidase products would be 

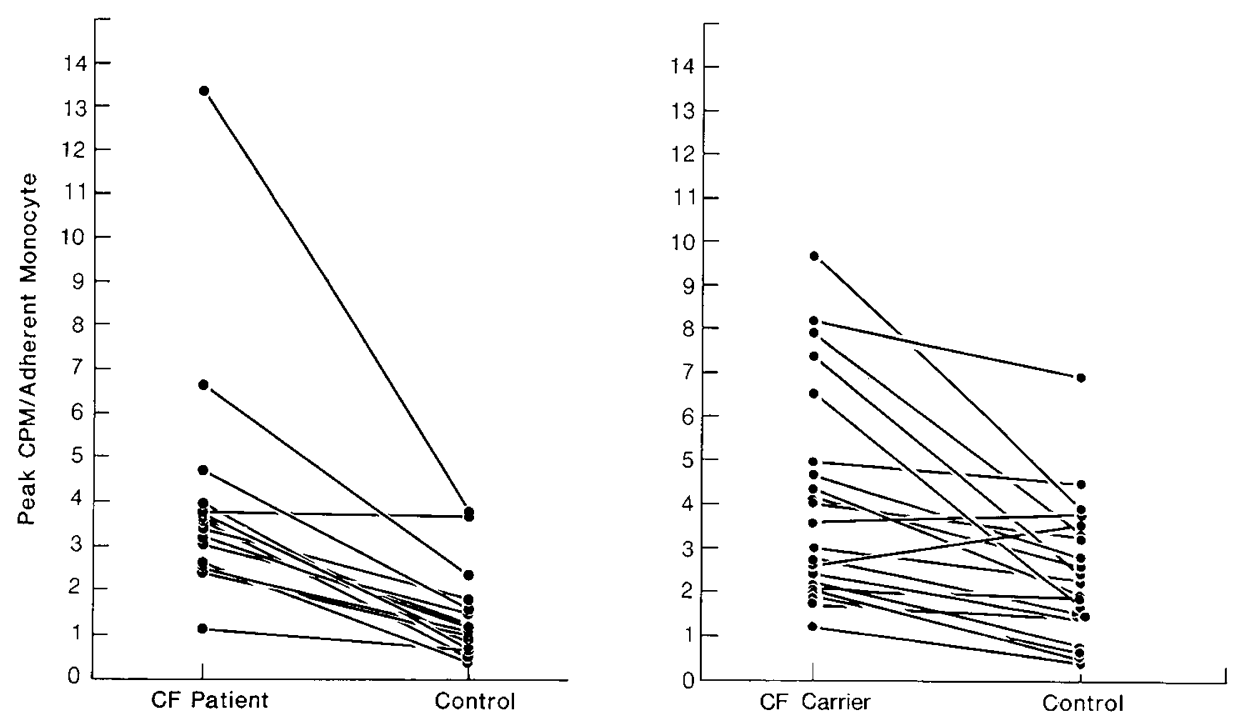

Fig. 2. Adherence-induced CL: each point represents the peak CPM/adherent monocyte for each CF patient connected to his/her age- and sexmatched control's value. Values are based on duplicate determinations after background CL was subtracted. Similarly, each parental carrier's peak CPM/adherent monocyte is connected to his/her age- and sex-matched control's value. For statistical analysis of the differences see Figure 3.

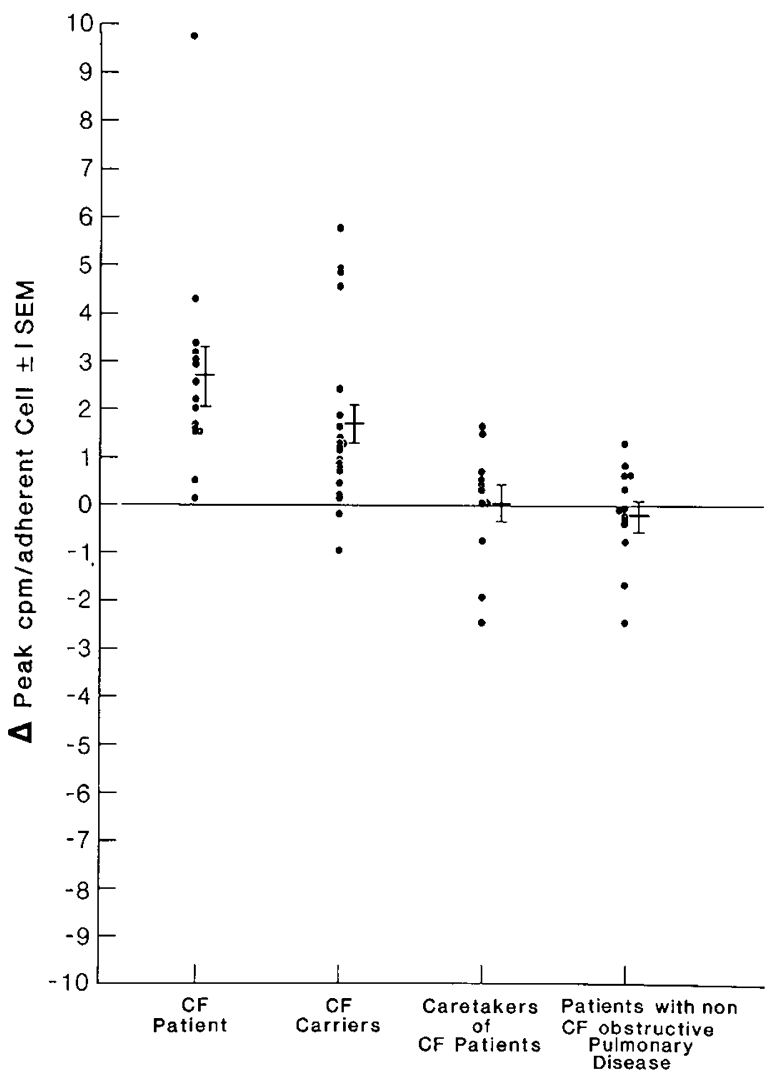

Fig. 3. Adherence-induced CL: each point is the difference between an individual's initial peak CPM/adherent monocyte in an experimental group and that of his age- and sex-matched control. Values are based on duplicate determinations and after background $\mathrm{CL}$ was subtracted. $\mathrm{CF}$ patients had a mean difference from control in peak $\mathrm{CL} /$ cell \pm 1 SEM of $2.70 \pm 0.57\left[p_{\alpha}(\mathrm{t})<0.001\right]$; CF carriers of $1.69 \pm 0.40\left[p_{\alpha}(\mathrm{t})<\right.$ $0.002]$. Caretakers of $C F$ patients who were not biologically related and non-CF patients with obstructive pulmonary disease showed mean differences from matched controls $0.02 \pm 0.39$ and $-0.20 \pm 0.26$, neither of which differ significantly from 0 . The mean of the differences in the carriers differed significantly from that of the caretakers $\left[p_{\alpha}(\mathrm{t})<0.001\right]$ and from that of patients with non-CF obstructive pulmonary disease $\left[p_{\alpha}(\mathrm{t})<0.001\right]$. products of arachidonic acid cyclooxygenase and endoperoxidation. This pathway has been shown to be active in CL generated by platelets on exposure to exogenous fatty acids (21). However, the efficiency of the interaction of these products with luminol to produce $\mathrm{CL}$ has not been defined and no exogenous fatty acids were added in our system. Our results therefore suggest that during the first $25 \mathrm{~min}$ after adherence to glass liquid scintillation vials, monocytes from $\mathrm{CF}$ patients and $\mathrm{CF}$ carriers either generated increased amounts of oxygen radicals when compared with normals or secreted more enzyme(s) such as peroxidase that would catalyze the generation of oxygen radicals. However, monocytes from CF carriers and normals generated essentially equivalent amounts of these putative oxygen radicals when stimulated with opsonized zymosan or PMA. This difference among differing stimuli could relate to differences in membrane-surface interaction, membrane receptor-surface interaction, ionic flux, second messenger stimulation, compartmentalization of the oxidases involved in the oxygen radical formation (22), or differences in the oxidase pathways. Further investigation by use of other stimuli, alternative luminogenic probes, and metabolic inhibitors is currently underway.

It should be emphasized that the CL difference between $\mathrm{CF}$ patients and controls and between CF carriers and controls were produced by monocytes that had been purified by adhering to preconditioned plastic flasks. It is possible that this step selected a particular monocyte subset and/or activated the surface membrane of these monocytes permitting the detection of these differences from control. However, since there was no difference in the application of this purification technique to any of the experimental or control groups, the technique itself cannot explain the differences in CL we have observed.

Our finding of increased CL of $\mathrm{CF}$ and $\mathrm{CF}$ carrier phagocytic cells is consistent with the finding that $\mathrm{CF}$ and $\mathrm{CF}$ carrier fibroblasts show increased $\mathrm{O}_{2}$ uptake and a variant NADH dehydrogenase (6), but we have no direct evidence at this time that this particular enzyme system is involved in the phenomenon we have observed. The significance of this early increase in presumed oxygen radical formation and/or release for host defense against microbes or for oxidative damage to host tissues remains to be defined.

The fact that the phenotypically normal parents of patients with $\mathrm{CF}$ showed an increase in adherence-induced $\mathrm{CL}$ and that the differences from control did not vary with age or length of exposure of a carrier to a child with $\mathrm{CF}$ argue strongly that this 
phenomenon is not simply secondary to either the medications or the respiratory dysfunction seen in the patients with $\mathrm{CF}$; the fact that patients with other types of obstructive lung disease did not show an increase also indicates that it may be unique to $\mathrm{CF}$. Moreover, repeated exposure to medications and respiratory secretions of CF patients was not associated with an increase in adherenced-induced $\mathrm{CL}$ in monocyte samples from their nonbiologically related caretakers nor did the biological parents of patients with $\mathrm{CF}$ show increasing adherence $\mathrm{CL}$ with increasing exposure time to the drugs and therapies prescribed for their children. We conclude that increased early phase adherenceinduced monocyte CL occurs in patients with CF and the obligate parental carriers of the $\mathrm{CF}$ gene independent of environmental influences.

Acknowledgments. The authors acknowledge the aid of Dr. Paul Hammon, Mrs. Marilyn Wilson, and Dr. Warren Warwick.

\section{REFERENCES}

1. Shwachmann H 1978 Cystic fibrosis. Curr Probl Pediatr 8:1-72

2. Lobeck CC 1972 Cystic fibrosis. In: Stanbury JB, Wyngaarden JB, Fredrickson DS (eds) The Metabolic Basis of Inherited Disease. McGraw-Hill, Inc., New York, pp 1605-1676

3. Di Sant Agnese PA, Davis PB 1976 Research in cystic fibrosis. N Engl J Med 295:481-485

4. Di Sant Agnese PA, Darling RC, Perara GA, Shea A 1953 Abnormal electrolyte composition of sweat. Pediatrics 12:549-563

5. Farber SJ 1945 Some organic digestive disturbances in early life. Mich State Med Soc J 44:587-594

6. Shapiro BL, Feigal RJ, Lam LEH 1979 Mitochondrial NADH dehydrogenase in cystic fibrosis. Proc Nat Acad Sci USA 76:2979-2983

7. Reiss M, Roos D 1978 Differences in oxygen metabolism of phagocytosing monocytes and neutrophils. J Clin Invest 61:480-488
8. Mills EL, Rholl KS, Quie PG 1980 Luminol-amplified chemiluminescence: a sensitive method for detecting the carrier state in chronic granulomatous disease. J Clin Microbiol 12:52-56

9. Stjernholm RL, Allen RC, Steele RH, Waring WW, Harris J 1973 Impaired chemiluminescence during phagocytosis of opsonized bacteria. Infect Immun 7:313-314

10. Grebner JV, Mills EL, Gray BH, Quie PG 1977 Comparison of phagocytic and chemiluminescence response of human polymophonuclear neutrophils. J Lab Clin Med 89:153-159

11. Babior BM 1978 Oxygen-dependent microbial killing by phagocytosis. N Engl J Med 298:659-668

12. Yanai M, Quie PG 1981 Chemiluminescence by polymorphonuclear leukocytes adhering to surfaces. Infect Immun 32:1181-1186

13. Gibsn LE, Cooke RE 1959 A test for concentration of electrolytes in sweat in cystic fibrosis of the pancreas utilizing pilocarpine by iontophoresis. Pediatrics 23:545-549

14. Brasfield D, Hicks G, Soong S-J, Peters J, Tiller R 1980 Evaluation of scoring system of the chest radiograph in cystic fibrosis: a collaborative study. Am $\mathrm{J}$ Radiol 134:1 195-1198

15. Ackermann SK, Douglas SD 1978 Purification of human monocytes on microexudate coated surfaces. J Immunol 120:1372-1374

16. Regelmann WE, Gray ED, Wannamaker LW 1982 Characterization of human cellular immune response to purified group A streptococcal blastogen A. J Immunol 128:1631-1636

17. Sbarra AJ, Karnovsky ML 1959 The biochemical basis of phagocytosis: Metabolic changes during the ingestion of particles by polymorphonuclear leukocytes. J Biol Chem 234:1355-1362

18. Allen RC Loose LD 1976 Phagocytic activation of a luminol-dependent chemiluminescence in rabbit alveolar and peritoneal macrophages. Biochem Biophys Res Commun 69:245-252

19. Allen RC 1982 Chemiluminescence and the study of phagocyte redox metab olism. Advan Exp Med Biol 141:411-42

20. Brestel EP 1985 Co-oxidation of luminol by hypochlorite and hydrogen peroxide. Implications for neutrophil chemiluminescence. Biochem Biophys Res Commun 126:482-488

21. Mills EL, Gerrard JM, Filipovich D, White JD, Quie PG 1978 The chemiluminescence response of human platelets. $\mathbf{J}$ Clin Invest 61:807-814

22. Hallett MB, Campbell AK 1983 Two distinct mechanisms for stimulation of oxygen-radical production by polymorphonuclear leukocytes. Biochem $\mathbf{J}$ 216:459-465 